\title{
Researcher Degrees of Freedom and a Lack of Transparency Contribute to Unreliable Results of Nonparametric Statistical Analyses Across SPSS, SAS, Stata, and $R$
}

Cooper B. Hodges*1,2, Bryant M. Stone*3, Paula K. Johnson²,5, James H. Carter III ${ }^{4}$, and Hannah M. Lindsey*1,2

${ }^{1}$ Department of Psychology, Brigham Young University, Provo, UT

${ }^{2}$ Department of Neurology, University of Utah, Salt Lake City, UT

${ }^{3}$ Department of Psychology, Southern Illinois University, Carbondale, IL

${ }^{4}$ Department of Psychology, Stanford University, Stanford, CA

${ }^{5}$ Neuroscience Center, Brigham Young University, Provo, UT

*These authors contributed equally and are considered co-first authors.

Corresponding author: Cooper B. Hodges, Department of Psychology, Brigham Young University, Provo, UT, 84602. E-mail: cooper.hodges@hsc.utah.edu 


\begin{abstract}
The replication crisis within the social and behavioral sciences has called into question the consistency of research methodology. A lack of attention to minor details in replication studies may limit researchers' abilities to reproduce the results. One such overlooked detail is the statistical programs used to analyze the data. In the current investigation, we compared the results of several nonparametric analyses and measures of normality conducted on a large sample of data in SPSS, SAS, Stata, and $R$ with results obtained through hand-calculation using the raw computational formulas. Multiple inconsistencies were found in the results produced between statistical packages due to algorithmic variation, computational error, and lack of clarity and/or specificity in the statistical output generated. We also highlight similar inconsistencies in supplementary analyses conducted on subsets of the data, which reflect realistic sample sizes. These inconsistencies were largely due to algorithmic variations used within packages when the analyses are performed on data from small- or medium-sized samples. We discuss how such inconsistencies may influence the conclusions drawn from the results of statistical analyses depending on the statistical software used, and we urge researchers to analyze their data across multiple packages, report details regarding the statistical procedure used for data analysis and consider these details when conducting direct replications studies.
\end{abstract}

Keywords: researcher degrees of freedom, reproducibility, transparency, nonparametric procedures, statistical software 


\section{Introduction}

Behavioral scientists are increasingly questioning the validity of using null hypothesis testing to distinguish the presence of an effect from random noise (Gelman \& O'Rourke, 2014). Concerning reports of questionable research practices (Giner-Sorolla, 2012; Head, Holman, Lanfear, Kahn, \& Jennions, 2015; Simonsohn, Nelson, \& Simmons, 2014) and a lack of replicability of highly-cited published research in the field (OSC, 2015; Ioannidis, 2014; Shrout \& Rodgers, 2018) are at the heart of this mistrust. Questionable research practices include flexible behaviors, such as stopping data collection once researchers achieve statistical significance, conducting numerous statistical comparisons with the intention of selectively reporting the statistically significant effects that support desirable outcomes (i.e., $p$-hacking), or hypothesizing after the results are known (HARKing; Kerr, 1998). Questionable research practices substantially increase Type-I error rates, misrepresent the scientific process by undermining the hypothetico-deductive model of the scientific method (see Figure 1), and are key contributors to the growing "replication crisis" (Ioannidis, 2005). Any scientific field risks losing credibility with a significantly high number of published false positive results (Simmons, Nelson, \& Simonsohn, 2011). These questionable research practices blur the lines between true and artifactual effects, which suggest a notable problem across behavioral science research.

[Insert Figure 1 approximately here]

Replication across several samples and researchers increases the legitimacy of a scientific claim; however, highly-cited scientific studies have demonstrated poor replicability (Ioannidis, 2014; Shrout \& Rodgers, 2018). In an attempt to replicate numerous studies published in high impact journals, the Open Science Collaboration (2015) demonstrated that only $36 \%$ of the studies replicated successfully, and the effect sizes found in the replications were approximately 
half of those in the original studies. These findings suggest a more widespread problem within the social and behavioral sciences: a knowledge base built on false positives. To address this concern, the scientific community is calling for transparency in research through open science, which may aid in both reducing the false positives published in the literature and increase reproducibility and replicability by encouraging ethical research practices (Ioannidis, 2014). Proponents of open science advise researchers to document and share a detailed account of their study procedures (including the experimental design, data collection process, and analysis procedures; Borghi \& Van Gulick, 2018), make their data available to the public, and preregister their studies. Some have further argued that researchers should note their study's researcher degrees of freedom (Wicherts et al., 2016), which refers to the choices the researchers make when preparing and analyzing the data (Simmons et al., 2011). Documenting researcher degrees of freedom may make it possible to distinguish between true and artificial replication failures by showing that significant findings occur only when another researcher uses the exact same choices (Epskamp, 2019).

Variability in the models used in statistical software packages is a critical determinant of researcher degrees of freedom. For the same analyses performed using various software packages, researchers have found differences in computational speed (McCoach et al., 2018), accuracy (McCullough, 2000; McCullough \& Heiser, 2008), and quantitative results (Bergmann, Ludbrook, \& Spooren, 2000; Keeling \& Pavur, 2007). Although relatively more advanced statistical software (e.g. $R$, Stata) offer some advantages, the increased complexity inherent to such packages lends to higher researcher degrees of freedom, which may increase the likelihood of unreliable results. We suggest that such discrepancies between statistical packages are overlooked in terms of their contribution to the replication crisis (see Figure 1). 


\section{Statistical Rigor}

Another requirement of open science is statistical rigor, which is defined as the "consistency in conceptual development, epistemological stance, application of analytical tools and transparent reporting of their use, and subsequent interpretation and reporting of findings" (Köhler, Landis, \& Cortina, 2017, p. 713). Two of the most common threats to statistical rigor are the inappropriate use of statistical analyses (Dar, Serlin, \& Omer, 1994; Garcia-Perez, 2012; Schatz, Jay, McComb, \& McLaughlin, 2005) and the misreporting and misinterpretation of results (Bakker \& Wicherts, 2011; Berle \& Starcevic, 2007). Additionally, there is evidence of incorrect statistical procedures (Nieuwenhuis, Forstmann, \& Wagenmakers, 2011), and reporting errors (Bakker \& Wicherts, 2011) in roughly 50\% of the published neuroscience and psychology literature, respectively. Maintaining statistical rigor ensures the appropriate and ethical use of statistical analyses that uphold the scientific method and contribute credible results to the scientific literature.

A fundamental principle of the scientific method is that reliability is necessary, but not sufficient, for validity (see Figure 2). This principle highlights the importance of reproducing results across various instruments, as one instrument may produce invalid results just as reliably as another produces valid results. This principal also applies to the reliability and validity of the tools used to conduct statistical analyses, specifically, the output of various statistical software packages commonly used within a field of research. Statistical reporting errors have been known to occur due to variations in rounding and reporting of $p$-values across software platforms (Levine \& Atkin, 2004), and the algorithms used to produce statistical output are often inconsistent between programs (Bergmann et al., 2000), particularly when conducting tests of nonparametric inference. 
[Insert Figure 2 approximately here]

Inappropriate statistical analyses lead to unreliable measures, or "instability" (Campbell, 1969), which threatens statistical conclusion validity, a special form of internal validity that concerns sources of random error and the appropriate use of statistics and statistical tests (Cook \& Campbell, 1979). Inappropriate test usage may occur when an analysis is unable to produce a logical or reliable answer to the research question (Garcia-Perez, 2012). Often, this is seen when traditional, non-robust procedures (e.g., independent $t$-test, ANOVA, regression) are used when assumptions, such as normality and homogeneity of variance, are violated. As a result, Type-I and Type-II error rates are uncontrolled and inferential results and effect sizes will be over- or underestimated (Osborne \& Waters, 2002). Ultimately, this leads to unreliable, and therefore invalid, results. These errors are a noteworthy problem within scientific literature, particularly in psychology, where any mention of checking assumptions is mentioned in roughly $8 \%$ of published studies (Hoekstra, Kiers, \& Johnson, 2012; Keselman et al., 1998; Osborne, 2008). Inappropriate test usage also occurs when the statistical analysis does not match the design of the study (Garcia-Perez, 2012). This error is simply due to a lack of understanding of statistical procedures, which arises from the widespread, longstanding lack of adequate training in statistics at the undergraduate and graduate level (Aiken, West, \& Millsap, 2008, 2009; Friedrich, Buday, \& Kerr, 2000; Rubin, Bell, \& McClelland, 2018). This problem is reinforced by an over reliance on point-and-click statistical software, which does not require any formal understanding of the underlying procedures (Borsboom, 2006; Garcia-Perez, 2012). Statistical methodology and subsequent quantitative results are fundamental components of the scientific method, as they provide the foundation for substantive scientific conclusions, future research questions, and meta-analytic input; therefore, it is of utmost importance to instill the importance 
of statistical rigor in students and practicing it when publishing the results of research in the social and behavioral sciences.

Reporting errors also threaten statistical rigor; however, lack of replicability due to reporting errors can be a consequence of differences in statistical software packages. Variations in rounding and reporting of $p$-values, and discrepant outputs due to differences in algorithms have been known to occur across different statistical software packages (Levine \& Atkin, 2004).

\section{Nonparametric Inference}

The use of nonparametric procedures provides a way to combat many of the problems described above. Their relative lack of reliance on assumptions and distribution-free methods protect researchers from making false conclusions that can result from misleading significance values obtained from parametric procedures with violated assumptions (Potvin \& Roff, 1993). There are several advantages to nonparametric methods, namely that they are distribution-free, and thus require few assumptions about the underlying populations from which the data are obtained. The basic principle of nonparametric inference is to estimate unknown quantities from data, while making as few assumptions as possible (Wasserman, 2006). Nonparametric analyses may be more robust and efficient than traditional normal-theory based procedures when the underlying populations are non-normal, and they offer greater statistical power than their parametric counterparts when sample sizes are small and when assumptions, particularly normality and homogeneity of variance, are violated (Hollander, 2014; Zimmerman \& Zumbo, 1993). Furthermore, under normal theory, nonparametric methods can be almost as robust and powerful as parametric methods (Siegel \& Castellan, 1988). Additionally, nonparametric procedures allow for exact statistics for $p$-values, confidence intervals, and experiment-wise error rates when the underlying populations are normal or non-normal; however, exact statistics 
are typically only available for small to medium sample sizes. Nonparametric statistics rely on the rank of values, rather than the magnitude; thus, they are relatively robust to outliers and can be used for the analysis of non-normal scales of measurement, such as ordered categorical data, in addition to continuous, interval or ratio data (Zimmerman \& Zumbo, 1993).

Disadvantages to using nonparametric methods exist as well, and the greatest of these is that they are geared toward hypothesis testing rather than effect estimation. Although it is possible to obtain nonparametric estimates of effect and associated confidence intervals, the processes by which this is done is not generally straightforward (Whitley \& Ball, 2002). Furthermore, nonparametric statistics are not robust when data are heteroscedastic (Erceg-Hurn \& Mirosevich, 2008; Zimmerman, 1998, 2000), and adjustments to nonparametric test statistics are necessary in the presence of tied values, which is common when the data are not continuous. In terms of their implementation, while critical value tables for probability distributions used for parametric analyses (e.g., $z, t, F)$ are commonly provided in textbooks and online resources, such resources are scarce for the majority of nonparametric statistics. Appropriate software for nonparametric methods is limited, and the way in which a software package deals with tied values, how it obtains appropriate $p$-values, and what specifications are used by default in a given software package are not always clear.

Algorithmic variations. The algorithms used to compute test statistics for rank- and frequency-based nonparametric tests depend on the options specified for at least three situations: (1) whether the exact, null distribution or a large-sample approximation of the normal $(z)$ or $\chi^{2}$ distribution is used; and when the asymptotic distribution (i.e., the large-sample approximation) is used, whether corrections for (2) continuity and/or (3) tied ranks are applied (Lehmann, 1998; 
Siegel \& Castellan, 1988). While these situations vary depending on the characteristics of the data, they may also vary due to researcher degrees of freedom.

Exact $p$-values and confidence intervals are calculated using the true underlying null distribution, which is discrete in most parametric inference; however, due to computational inefficiency and a lack of null distribution tables for samples of approximately $n>30$, an approximation of the true distribution is typically used to calculate asymptotic probabilities for large samples. Asymptotic distributions rely on the central limit theorem, assuming that the sample is large enough so that the test statistic approaches the normal distribution. Parameters that do not assume a normal distribution, such as rank-ordered nonparametric tests, are often estimated using asymptotically distribution-free methods that evaluate the median, rather than the mean (Huang \& Bentler, 2015; Neave \& Worthington, 1988). Exact and asymptotic $p$-values are very similar when obtained from large samples, but they can be quite different when sample sizes are small; thus, asymptotic $p$-values may lead to unreliable and misleading conclusions if used inappropriately.

When asymptotic distributions are continuous (e.g., normal, $\chi^{2}$ ), continuity correction procedures may be applied to improve approximations of the underlying discrete null distribution of nonparametric procedures (Gibbons \& Chakraborti, 2011). Controversy exists regarding the use of continuity correction procedures (Conover, 1974; Maxwell, 1976); in particular, it has been argued that continuity correction is only appropriate for approximating test statistics and $p$ values of one-sided tests (Haber, 1982; Mantel, 1976). When applied to two-sided tests, continuity corrections are systematically conservative, resulting in over-corrected (larger) $p$ values (Maxwell, 1976; Stefanescu, Berger, \& Hershberger, 2005). Presently, textbooks and 
software packages are inconsistent in their recommendation and default use of continuity correction procedures (Hitchcock, 2009).

Rank-ordered statistics are typically assumed to be drawn from a continuous population, of which the probability of any two observations being equal in magnitude is zero (Gibbons \& Chakraborti, 2011; Siegel, 1957). In practice, two or more observations of the same magnitude commonly occur due to measurement imprecision or because the population distribution is actually discrete. Such observations are considered to be tied, and some method of assigning unique ranks to tied values must be applied so that the test statistics that depend on relative magnitudes of observations (i.e., rank-order statistics) can be computed (Gibbons \& Chakraborti, 2011). Most commonly, individual observations within a group of tied observations are assigned the average of the group's ranked value. Although this method does not affect the mean ranked value, it reduces the variance of the ranks. This affects the underlying null distribution; thus, a correction for ties is frequently applied to the calculation of the test statistic.

Typically, the default algorithms used within a statistical software package depends on the sample size and the presence or absence of tied ranks. As a result, different procedures may be applied to datasets of variables with different properties. When the default algorithmic specifications are not made clear in statistical output, non-statisticians are unlikely to know if correction procedures were applied to asymptotic $p$-values or if the probability statistics reported are exact. This can lead to the use of inappropriate procedures and reporting of statistical errors or misleading results. Furthermore, because default algorithms vary across statistical packages, the results reported by researchers who are naïve to the specific algorithms used to compute them might have differed if another statistical package was used; thus, the conclusions made from 
statistical analyses conducted under such conditions may be mere reflections of the software used rather than the actual data analyzed.

\section{Rationale and Objective}

We see a gap in previous research literature on psychological methods, as there are few published studies that methodically document the differences produced between statistical software programs. Although some previous work has demonstrated the formulaic and computational differences in the calculation of certain parametric and nonparametric methods (Bergmann et al., 2000; Brown, Hendrix, Hedges, \& Smith, 2012; McCoach et al., 2018; Oster \& Hilbe, 2008a, 2008b; Wang \& Johnson, 2019), this work is largely geared toward mathematicians and statisticians, resulting in considerable difficulty in generalization to various subfields within the social and behavioral sciences. The present study seeks to systematically document the inconsistencies in results that different software packages produce when conducting the same nonparametric analyses on the same dataset. We believe that these disparities are important for other researchers to be aware of when conducting statistical analyses.

The aim of the present methodological investigation was to evaluate the reliability of the statistical output from various nonparametric tests and two measures of normality, using default parameters and as few additional specifications/options as possible, across four statistical software packages commonly used in the social and behavioral sciences: SPSS, SAS, Stata, and $R$. We conducted this investigation using a large, public dataset $(n=414,167)$ that is widely used in the social psychology literature. We hypothesized that the results of all analyses would be largely consistent across the statistical platforms, although minor discrepancies were expected at the decimal level due to rounding. Larger discrepancies in the results were expected to occur 
with increasing levels of researcher degrees of freedom related to the number of specifications required to conduct each analysis using a given algorithmic procedure.

Given that the majority of published psychological science research is conducted using much smaller samples, we decided to conduct two post-hoc exploratory investigations on subsets of the original dataset with medium and small samples of $N=100$ and $N=30$, respectively. After reviewing the results of the initial, Large $N$ analyses and determining the sources of the discrepancies that were found between the output of the four statistical packages for each test, we explored the extent to which the level of inconsistency seen in the results of the Large $N$ analyses would be present in the results of Medium and Small $N$ analyses.

\section{Disclosures}

The raw datasets (consisting only of the variables used in the present study), the scripts or syntax used for all analyses (including the MS Excel spreadsheets used for hand-calculations), all relevant help files/documentation provided by each software package, and logs of the full statistical output generated from each software platform can be found in our OSF storage directory at https://osf.io/35umb/. This study involved the analysis of existing data from Project Implicit (Xu, Nosek, \& Greenwald, 2014) rather than new data collection. The original Race IAT Public 2018 dataset was downloaded on June 06, 2019 from the data archives hosted on the Project Implicit web́site (https://projectimplicit.net); however, the Project Implicit data archives have since been relocated to https://osf.io/z4bd2/, where the raw Race IAT Public dataset and corresponding codebook can be accessed directly. It is unknown whether any data loss/corruption may have occurred during this transition, thus the raw dataset we downloaded prior to the relocation of the Project Implicit data archives, which was used in the present 
analyses, can also be accessed from our OSF storage directory. This manuscript was posted to the PsyArXiv preprint archive prior to submission.

\section{Method}

\section{Dataset}

For the current investigation, the open-source Race Implicit Associations Test (IAT) 2018 data (Xu et al., 2014) was used for all analyses. The Race IAT was designed to assess for underlying attitudes toward white and black people that participants may be unwilling or unable to identify within themselves (Lane, Banaji, Nosek, \& Greenwald, 2007). Project Implicit hosts the Race IAT data for public use (https:/osf.io/gwofk/) and allows researchers access to the data freely. We chose this dataset due to its wide-spread use within the social and behavioral sciences (e.g., 12,900 results were returned for a single Google Scholar search for "Implicit Association Test" AND "Race" on February 12, 2020).

We selected eight variables (see Table 1) from the Race IAT Public 2018 dataset based on the relevance of their scale of measurement and number of levels for nonparametric analyses, the fewest number of missing data points, and the likelihood of being used in the experimental research published in the literature. It is important to emphasize that the variables used presently were not chosen for their underlying measured constructs, thus the results of our analyses are not meant for conceptual interpretation; rather, the variables used in the present study are solely meant for the quantitative comparison of results across statistical software packages.

[Insert Table 1 approximately here]

The raw Race IAT Public 2018 dataset consists of 460 variables and 859,470 observations. Data cleaning was performed using Stata version 16.0 (StataCorp LLC, College Station, TX), and this initially involved dropping all observations from respondents who did not 
complete the Race IAT (session_status values != "C") in addition to 1,290 observations, which were dropped for respondents who failed to complete all 120 trials across the four combined-task blocks $($ N_3467 values $<120)$. The remaining 414,167 observations were included in the final, Large $N$ dataset used in the present analyses.

\section{Nonparametric Statistics}

The decision to focus on nonparametric procedures was determined through the consensus of all authors for the following reasons: (1) nonparametric analyses are commonly used in the literature to compare sample characteristics between participant groups (e.g., Pearson's chi-square test of independence), although less commonly for various other analyses; (2) while hand- and computer-based calculations of common parametric analyses are typically covered in undergraduate and graduate statistics courses, nonparametric analyses are rarely or never covered to the same degree, especially in required statistics courses (Alder \& Vollick, 2000; Friedrich, Childress, \& Cheng, 2018). This may lead to an increased reliance on statistical software for computing such analyses and a decreased likelihood that errors in computation will be found or that variations in default algorithms used by different software platforms will be noticed; (3) other researchers have recently published material comparing statistical software programs for common parametric analyses as well as more complex statistical models (Bergmann et al., 2000; Brown et al., 2012; McCoach et al., 2018; Oster \& Hilbe, 2008a, 2008b; Wang \& Johnson, 2019); (4) the authors felt confident in their own understanding and in the capacity of the software programs used in the present study to perform the chosen nonparametric analyses.

Present analyses. We compared the results of five commonly used nonparametric tests, including Pearson's chi-square test for independence, Spearman's rank-ordered correlation (rho), 
the Kruskal-Wallis one-way analysis of variance, the Wilcoxon-Mann-Whitney U rank-sums test, and the Wilcoxon signed ranks test. Each of these nonparametric tests are described in Table 2. Because it is often necessary to evaluate normality when deciding whether or not parametric tests are inappropriate, we also compared calculations of skewness (the degree to which a set of data are symmetrically or asymmetrically distributed around the mean) and kurtosis (the extent to which the peakedness of a probability distribution deviates from the shape of a normal distribution) across software platforms.

[Insert Table 2 approximately here]

\section{Documentation of Procedure}

The statistical software packages used include the following: SPSS 26.0 (IBM Corporation, LLC, Armonk, NY, 2019), SAS JMP Pro 15.0 (SAS Institute Inc., Cary, NC, 2019), Stata 16.0 (StataCorp LLC, College Station, TX, 2019), and $R$ 3.6.0 (The $R$ Foundation, 2016). All code used to generate results in command-line-based programs (Stata, $R$ ) were documented, and in the case of programs with a graphical user-interface (SPSS, SAS), the underlying syntax generated from the point-and-click commands were also documented.

Prior to comparing the results across packages, we calculated each test statistic and asymptotic $p$-value with and without corrections for continuity and ties (when applicable) using the raw, computational formulas (see Supplementary Material), and the results were considered the standard by which all results generated from statistical packages were compared. Due to the large size of the dataset used, literal hand-calculations were not possible. Rather, we performed these calculations in MS Excel for Mac version 16.32 (Microsoft Corporation, Redmond, WA, 2019) and henceforth refer to these results as those derived by hand-calculation. When applicable, all analyses were conducted using two-tailed alphas. 


\section{Criteria for Meaningful Differences in Results}

We used the following criteria to determine what would be considered "meaningful" differences in results produced across packages: inconsistencies due to (1) algorithmic variation (e.g., no correction, adjustment for ties, and/or continuity correction), (2) computational error (i.e., results differ from those obtained through hand-calculation despite the use of the same computational procedure), or (3) statistical output (e.g., reporting of exact versus asymptotic $p$ value; reporting a $p$-value without reporting the test statistic). The latter criteria was included due to the assumption that the same statistics should be reported across software programs, and the use of any variations in the calculation of a test statistic or $p$-value should be made explicitly clear in the output and provided along with traditional (i.e., uncorrected) calculations.

\section{Supplementary Analyses}

To assess if any of the inconsistencies present in the results of our Large $N$ analyses were present in smaller samples that more closely reflect those used in the psychological science literature, we conducted the same analyses in the same programs on subsets of data taken from our Large $N$ dataset as supplementary analyses. For these supplementary Medium $N$ and $\operatorname{Small} N$ analyses, we selected the first 100 and 30 cases with no missing data. The same scripts/syntax and hand-calculation procedures used in the Large $N$ analyses were applied to the two supplementary analyses; however, fewer steps were required to calculate the test statistics by hand, as there were no missing data and fewer analyses requiring a correction for ties.

\section{Results}

In the following sections, we describe the default procedures and algorithms used by SPSS, SAS, Stata, and $R$ to conduct the following analyses: Pearson's chi-square test of independence, Spearman's rank correlation coefficient (rho), Kruskal-Wallis one-way analysis of 
variance, Wilcoxon Mann-Whitney U rank sums test, Wilcoxon signed ranks test, skewness, and kurtosis. Additionally, we provide details regarding the hypothesis tested, according to the respective package's help files/documentation, the statistical output generated from each analysis conducted across statistical packages, and additional options or arguments that must be specified by the user (i.e., are not applied by default) in order to generate specific results (e.g., test statistic, exact $p$-values) or to use alternative algorithms (e.g., apply a continuity correction) to compute a test statistic. Prior to reporting the results obtained through statistical software, we provide a description of the various hand-calculations conducted to obtain asymptotic test statistics (with and without corrections for ties and/or continuity, when applicable).

The numerical results of all analyses are reported in Table 3 for comparison across all statistical packages with the hand-calculated results. The results in Table 3 are organized according to the default algorithms used by a given package (i.e., asymptotic test statistics with or without correction for ties and/or continuity). Due to the large sample size used presently, exact $p$-values were neither generated by hand nor by any statistical package for the Large $N$ analysis.

\section{[Insert Table 3 approximately here]}

\section{Pearson's Chi-square}

Hand-calculation. To obtain asymptotic test statistics, $2 \times 2$ observed and expected frequency tables were generated, and the resulting values were used to obtain the degrees of freedom, $\chi^{2}$ test statistic, and a right-tailed, asymptotic $p$-value with and without Yate's continuity correction.

SPSS. Tests the hypothesis that the rows and columns in a two-way table are independent. The output includes a case processing summary of the number and percentages of 
valid, missing, and total cases, as well as a two-way contingency table, which includes cell, margin, and grand total observed frequencies (although not reported by default, there is an option available to specify for expected counts and percentages to also be generated). The output also includes a test statistic table, with $\chi^{2}$ values, degrees of freedom, and asymptotic two-tailed $p$ values for Pearson's chi-square test with and without Yates continuity correction, as a likelihood ratio, and for Mantel-Haenszel's test of linear association. When $2 \times 2$ tables are used, Fisher's exact one- and two-tailed $p$-values, which are reduced to the classical McNemar statistic (McNemar, 1947), are also provided.

SAS. Tests the hypothesis that the distribution of one categorical variable is equal across each level of the other variable. The output includes a graphical representation of the frequency table as a mosaic plot, followed by the two-way contingency table, which includes cell, margin, and grand total frequencies (observed), percentages of cell counts and margin totals to the grand total, percentages of each cell count to its respective row and column totals, and $\chi^{2}$ values computed for each cell. The output additionally includes a Test report, where the total number of observations, degrees of freedom, negative log-likelihood, and RSquare (U) values are reported, along with $\chi^{2}$ test statistics and asymptotic $p$-values for the likelihood ratio and Pearson's chisquare test of independence. When both variables have only two levels, Fisher's exact probabilities and alternative hypotheses are reported for one- (left and right) and two-tailed tests. No continuity correction is applied to the calculation of the $\chi^{2}$ test statistic by default, however this is not specified in the output.

Stata. Tests the hypothesis that the rows and columns in a two-way table are independent. The output includes an observed frequency table (although not reported by default, an option is available for expected frequencies to be reported), the $\chi^{2}$ test statistic, degrees of 
freedom, and an asymptotic $p$-value; there is no option available for the application of a continuity correction.

$\boldsymbol{R}$. Tests the hypothesis that the distribution of the cell frequencies in a two-dimensional contingency table is the product of the column and row marginal values. By default, Yates continuity correction is applied (although the user can specify that it is not applied), and this is clearly stated above the results generated in the output. The statistical output includes the $\chi^{2}$ test statistic, degrees of freedom, and asymptotic, two-tailed $p$-value.

\section{Spearman's rho}

Hand-calculation. Spearman's rank correlation coefficient (rho) and a two-tailed, asymptotic $p$-value was generated after applying a correcting for ties. A table of tied ranks was also generated, where unique values and the corresponding number of ties are provided for each variable.

SPSS. Tests the hypothesis that the correlation between the two rank-ordered variables is different from zero. The default algorithm used by SPSS corrects for ties, the formula used to calculate the correlation coefficient follows the procedure of Siegel (1956), and significance is determined based on the $t$-distribution with $N-2$ degrees of freedom. By default, missing data is handled case wise, although the user can specify that missing data is handled in a pairwise fashion. The output includes Spearman's rho correlation coefficients between the two variables of interest and for each variable with itself, each with a two-tailed $p$-value (although the user can specify that a one-tailed $p$-value is provided) and the number of observations included in the correlation.

SAS. Measures the strength of the relationship between two ranked variables using the formula for the Pearson's product-moment correlation, where tied values are assigned average 
ranks, by default. The output includes a correlations report, with a matrix of Pearson's $r$ correlation coefficients, the number of missing values, and a statement that the correlations are estimated using the pairwise method. A Nonparametric: Spearman's rho report is also provided in the output, which includes the names of the two variables analyzed as well as Spearman's rho correlation coefficient, the corresponding two-tailed $p$-value, and a color-mapped cell plot that displays the strength of the association on a scale from blue $\left(r_{\mathrm{s}}=-1.00\right)$ to red $\left(r_{\mathrm{s}}=+1.00\right)$.

Stata. Tests the hypothesis that the two variables are independent of each other. The output includes the number of observations, the value of Spearman's rho, and the two-tailed $p$ value. The calculation of the correlation coefficient is determined using case wise deletion, where cases are ignored if data from either of the variables are missing. The user may specify that pairwise deletion of observations with missing values should be used instead.

$\boldsymbol{R}$. Tests whether the rank-based association between paired samples is equal to zero. Ties are adjusted by default, and although no continuity correction is applied by default, the user may specify to do so. The output from $R$ includes an "S" test statistic [which is defined as $\left(n^{3}-n\right)(1-r) / 6$, where $n$ is the length of the vector, $x$, and $r$ is the Pearson correlation coefficient], an asymptotic two-tailed $p$-value based on the $t$-distribution, and the Spearman's rho correlation coefficient. For samples of $n<1290$, and when no ties are present, exact $p$-values are computed, by default.

\section{Kruskal-Wallis}

Hand-calculation. To obtain asymptotic test statistics, a frequency table including the rank sums and average squared rank sums was generated. Additionally, the degrees of freedom, Kruskal-Wallis $H$ test statistics (following the $\chi^{2}$ distribution), and right-tailed, asymptotic $p$ values with and without a correction for ties were calculated. To determine the tie correction 
value $\left(C_{H}\right)$, a second table was generated, which includes a list of all unique values with the corresponding number of ties, as well as a range of the number of tied values present in each set of tied values.

SPSS. Tests the hypothesis that two or more samples on a non-normal or ordinal dependent variable are from the same population distribution using ranks. The default algorithm applies a correction for ties. The output includes a table of the number of observations and mean ranks for each level of the grouping variable as well as the total number of observations, and a second table includes the Kruskal-Wallis $H$ test statistic, degrees of freedom, and asymptotic, one-tailed $p$-value.

SAS. Tests whether means or medians of ranked responses are equal across all levels of the grouping variable. Corrections for ties and continuity are applied by default. The output includes a one-way plot of responses across all levels of the grouping variable before a Rank Sums report, which includes a table that lists the number of observations, rank sums, expected scores, mean rank sums, and standardized ranked scores for each level of the grouping variable. The results of a one-way test for location using the $\chi^{2}$ approximation are also reported, which includes the value of the $\chi^{2}$ statistic, the degrees of freedom, and a one-tailed asymptotic $p$-value. Additionally, the output includes a statement of the number of rows with missing data.

Stata. Tests the hypothesis that several samples are drawn from the same population. The output includes a table with the number of observations and the rank sum for each level of the grouping variable, as well as $\chi^{2}$ test statistics, degrees of freedom, and one-tailed asymptotic $p$ values, each calculated with and without a correction for ties.

$\boldsymbol{R}$. Tests whether the location of the sample distributions are the same using rank-ordered data. By default, no correction for continuity or ties is applied. The output includes the value of 
the Kruskal-Wallis $\chi^{2}$ statistic, the degrees of freedom, and a one-tailed $p$-value, which is not specified as exact or asymptotic in the output or documentation.

\section{Wilcoxon Mann-Whitney U}

Hand-calculation. To obtain asymptotic test statistics, we generated a frequency table, including the number of total observations and rank sums observations for each level of the grouping variable, as well as the mean and variance of Wilcoxon $W$ statistic, and the mean and standard deviation of the rank sums for the smaller of the two groups. Additionally, a second table was generated to aid in the tie correction, which includes a list of all unique values with the corresponding number of ties. Finally, the following test statistics were calculated after correcting for ties, both with and without applying a continuity correction: Wilcoxon $W$, MannWhitney $U, z$-score, and a two-tailed, asymptotic $p$-value.

SPSS. Tests the hypothesis that two samples on a non-normal or ordinal dependent variable are from a population with the same distribution using ranks. A correction for ties is applied by default. The default output includes a table with the number of observations, mean ranks, and sum of ranks for each of the two groups, along with the Mann-Whitney $U$ test statistic (the smaller of two values, which are calculated as the number of times a score from either group precedes a score from the other group), the Wilcoxon $W$ test statistic (the sum of the ranks for the group with the smaller mean rank), a $z$-score test statistic, and a two-tailed, asymptotic $p$-value. By default, the exact two-tailed $p$-value is also reported for samples of $n \leq 400$, using the algorithm provided by Dineen and Blakesley (1973).

SAS. Tests whether means or medians of ranked responses are equal across both levels of the grouping variable. Corrections for ties and continuity are applied by default. The output includes a one-way plot of responses across all levels of the grouping variable before a Rank 
Sums report, which includes a table that lists the number of observations, ranks sums, expected scores, mean rank sums, and standardized ranked scores for each level of the grouping variable. The results of a two-sample test, using the normal approximation are also reported, including the rank sum (labeled as "S") for the observations in the smaller of the two groups (or the second level of the grouping variable, when there are an equal number of observations between groups), the test statistic approximating the normal distribution (z-score), and a two-tailed, asymptotic $p$ value. Exact statistics are available for the Wilcoxon Mann-Whitney U in SAS JMP Pro only (i.e., not available using SAS or JMP), using the Nonparametric $>$ Exact Test function. As in the output of the Kruskal-Wallis test, the results of a one-way test for location using the $\chi^{2}$ approximation are reported in a 1-Way Test, Chi-square Approximation report, which includes the $\chi^{2}$ test statistic, degrees of freedom, and an asymptotic $p$-value. Lastly, the output includes a statement of the number of rows with missing data.

Stata. Tests the hypothesis that two independent samples are from populations with the same distribution. The output includes a table with the number of observations, the sum of ranks, and the expected values for each level of the grouping variable, as well as a report of the unadjusted variance, the adjustment for ties, and the resulting adjusted variance used to calculate the test statistic. A $z$-statistic, based on Fisher's (1935) principle of randomization, is reported along with a two-tailed, asymptotic $p$-value. An exact $p$-value is also provided, by default, for total sample sizes of $n \leq 200$, although the user can specify that the exact $p$-value is generated along with the asymptotic $p$-value for samples of $n \leq 1000$.

$\boldsymbol{R}$. Tests whether the sample distributions differ by a location shift of $\mu$. An adjustment for ties and a continuity correction are applied by default, and the latter is clearly stated in the output. The output includes the $W$ test statistic, which represents the sum of ranks for the first 
sample with the minimum value subtracted, and a two-tailed, asymptotic $p$-value. Exact $p$-values can be specified for samples of $n<50$ when no ties are present; they are calculated using the algorithm described in Bauer (1972), and the Hodges-Lehmann estimator is employed.

\section{Wilcoxon Signed Ranks test}

Hand-calculation. To obtain asymptotic test statistics, we generated a frequency table including the number of observations and rank sums observations for the positive and negative ranks, as well as the number of zero differences, the number of ties, and the mean and standard deviation of Wilcoxon's $T$-statistic (based on the positive ranks). Due to a lack of tied ranks, no table of tied values was produced for this analysis. Finally, the following test statistics were calculated both with and without applying a continuity correction: Wilcoxon $T, z$-statistic, and a two-tailed, asymptotic $p$-value.

SPSS. Tests that the median of the distribution of the matched pairs of observations are different from zero. By default, the algorithm applies corrections for ties and zero differences. The output includes a table with the number of observations, mean ranks, and sum of ranks for positively and negatively ranked differences, the number of zero differences (which are inappropriately labeled in the table as "ties"), and the total number of observations. The output additionally reports a $z$-statistic with a clear indication that it is based on the positive ranks and a two-tailed, asymptotic $p$-value; no exact $p$-value is reported for samples of any size.

SAS. Tests if a single population of paired differences are symmetric around 0 by comparing the sizes of the positive differences to that of the negative differences. By default, zero differences are adjusted using the method suggested by Pratt (Cureton, 1967; Lehmann \& D’Abrera, 2006; Pratt, 1959), tied values are assigned average ranks, and no continuity correction is applied. The output includes a Difference report, with a plot of paired differences 
by paired means, and a table of values for the mean of each response, the mean, standard error, and $95 \%$ confidence interval for the difference, the total number of paired observations, and Pearson's $r$ correlation coefficient, as well as a $t$-statistic, degrees of freedom, and one- and twotailed $p$-values for a paired-samples $t$-test. Finally, a Wilcoxon Signed Rank report includes the value of the test statistic (labeled as "S"), based on the sum of absolute value of signed ranks, along with one- and two-sided asymptotic $p$-values, which are based on a Student's $t$ approximation (Iman, 1974; Lehmann \& D’Abrera, 2006). Exact $p$-values are generated, by default, for samples of $n<20$.

Stata. Tests the equality of matched pairs of observations, where the null hypothesis is that both distributions are the same; that is, that the distribution of matched pair differences has a median equal to zero, and it is assumed that this distribution is symmetrical. The output includes a table with the number of observations, the rank sums, and the expected values for pairs with positive, negative, and zero differences, and for the total number of pairs. Additionally, a report of the unadjusted variance, the adjustment for ties, the adjustment for zero differences, and the resulting adjusted variance used to calculate the test statistics is provided. As in the WilcoxonMann-Whitney $\mathrm{U}$ test, a $z$-statistic is reported along with the two-tailed, asymptotic $p$-value. An exact $p$-value is also reported, by default, for samples of $n \leq 200$, and the user may specify that the exact $p$-value is reported for samples of $n \leq 2000$.

$\boldsymbol{R}$. Tests whether the distribution of paired differences is symmetric around $\mu$. By default, a continuity correction and an adjustment for ties are applied, the former of which is clearly stated in the output. The output also includes the Wilcoxon $T$ test statistic(labeled as "V") and a two-tailed, asymptotic $p$-value. Exact $p$-values can be specified for samples of $n<50$ when no 
ties are present; they are calculated using the algorithm described in Bauer (1972), and the Hodges-Lehmann estimator is employed.

\section{Skewness and Kurtosis}

Hand-calculation. Computational formulas were used to determine the population skewness (Cramér, 1946), sample skewness (Bliss, 1967), population kurtosis, where the mean kurtosis of the normal distribution is equal to three (Bock, 1975), and excess sample kurtosis, where the kurtosis of the normal distribution is corrected to have a mean equal to zero (Cramér, 1946).

SPSS. Skewness is estimated using the unbiased sample formula provided by Bliss (1967), and kurtosis is estimated using the unbiased calculation of excess sample kurtosis, where the normal distribution is assumed to have kurtosis equal to zero. By default, the output includes the number of valid and missing observations and the values and standard errors of both skewness and kurtosis.

SAS. When estimating skewness and kurtosis, the output includes a vertical histogram, a Quantiles report with the values of various quantiles between $0 \%$ (minimum) and 100\% (maximum), and a Summary Statistics report, which includes values for the mean, standard deviation, standard error of the mean, $95 \%$ confidence interval around the mean, the total number of observations, and estimates of sample skewness (Bliss, 1967) and excess sample kurtosis (Cramér, 1946).

Stata. Skewness and kurtosis are estimated in Stata using basic approximations of population skewness (Cook \& Campbell, 1979; Cramér, 1946) and population kurtosis, where kurtosis of the normal distribution is equal to 3 (Bock, 1975). Stata does not provide any other option for the way in which skewness or are calculated; however, Stata users who wish to find 
sample estimates of skewness and/or excess kurtosis using user-specified formulas from Joanes and Gill (1998) may download the free, web-based Stata package, moments2.

$\boldsymbol{R}$. There is no built-in functionality for calculating skewness or kurtosis in $R$; however, web-based packages (e1071, moments) can be downloaded for free to estimate these measures of normality using user-specified formulas from Joanes and Gill (1998).

\section{Meaningful Differences Across Packages}

Algorithmic variation. The default algorithms used to produce the results of all analyses, with the exception of Spearman's rho, differ widely across the four packages we compared. When conducting Pearson's chi-square test, SAS and Stata apply no continuity correction, $R$ applies Yates continuity correction, and SPSS generates results both with and without Yates continuity correction. Although no ties were present in the variables assessed by the Kruskal-Wallis test, SAS and $R$ do not adjust for ties by default, whereas SPSS adjusts for ties, and Stata produces results both with and without an adjustment for ties. When performing the Wilcoxon Mann-Whitney $\mathrm{U}$, all packages adjust for ties; however, SAS and $R$ also apply a continuity correction, by default, whereas SPSS and Stata do not. Similarly, while all packages adjust for ties when performing the Wilcoxon Signed-ranks test, only $R$ also applies a continuity correction, by default. Additionally, the algorithm used to calculate the Wilcoxon $T$ statistic in SAS differs from the generally accepted definition provided by Wilcoxon (1945), where test statistic is the smaller of either the sum of ranks with positive differences or the sum of ranks with negative differences. While this definition is used by SPSS, Stata, and $R$, the test statistic produced by SAS is based on the sum of absolute value of signed ranks. Finally, unbiased calculations of sample skewness and excess sample kurtosis (where the expected value for kurtosis of a sample with a normal distribution is 0) are only used by SPSS and SAS. Despite the 
bias inherent in the use of population estimates, Stata does not provide any other built-in option for the way in which these measures of normality are estimated for a sample of data.

Additionally, it is important to note that there is no built-in function for calculating skewness or kurtosis in $R$.

The same algorithmic variations were applied to the computation of results when these analyses when they were performed for our Supplementary Medium $N$ (Table 4) and Small $N$ (Table 5) analyses, with two exceptions in the results of the Small $N$ analyses. The first appeared in the computation of exact $p$-values for the Wilcoxon Mann-Whitney U test by SPSS and Stata, where SPSS uses the algorithm provided by Dineen and Blakesley (1973), and Stata uses a recursive algorithm described by (Hill \& Peto, 1971); as a result, the exact $p$-values produced by SPSS was approximately .0061 greater than that which was produced by Stata. The second additional algorithmic variation appeared in the results of the Wilcoxon Signed-rank test when conducted on the Small $N$ dataset in $R$. For Spearman's rho, the Wilcoxon Mann-Whitney U, and the Wilcoxon Signed-Ranks test, $R$ automatically computes and reports the exact $p$-value in place of the asymptotic $p$-value if the sample size is $n<1290$ (Spearman's rho) or $n<50$ (Wilcoxon tests) and no ties are present. Due to the presence of ties in the data used for Spearman's rho and the Wilcoxon Mann-Whitney U test, the asymptotic $p$-values were reported along with a warning message stating that the exact $p$-value cannot be computed with ties. In contrast, there is no clear indication that the exact $p$-value was reported in place of the continuity-corrected asymptotic $p$ value in the output generated from the Wilcoxon Signed-ranks test in $R$; rather, the user must refer to the help files to be made aware of this.

[Insert Tables 4 and 5 approximately here] 
Computational error. We found several inconsistencies between the hand-calculated results and those produced by the statistical packages when the same computational procedures were used for calculation that could not be reduced to rounding error; we therefore determined that such inconsistent results were produced in error. Such errors were found for the results of Spearman's rho provided by SAS, Stata, and $R$ : although the results were consistent across these three packages, the values of Spearman's rho were all at least .0001 lower than that which was obtained through hand-calculation. It is of note that the results from SPSS are rounded to the third decimal place, and we were therefore unable to determine if the same error occurred. Errors were also present in the results of the Kruskal-Wallis test produced by Stata, where the values of the test statistic differed by -0.026 than those obtained through hand-calculation, and the $p$ values were reported as equal to .0001 , whereas those obtained through hand-calculation were less than .0001 . Additionally, the Mann-Whitney U statistic produced by SPSS was approximately $13^{\mathrm{e}+10}$ less than the value obtained through hand-calculation. Finally, the signs of the $z$-statistics produced for the Wilcoxon Mann-Whitney U by SPSS and for the Wilcoxon Signed-Ranks test by Stata were opposite to those of the $z$-scores obtained through handcalculation.

Two errors were found in the results of the Medium $N$ analyses, and both occurred in the computation of asymptotic $p$-values for the Wilcoxon Signed-Ranks test. The documentation provided by SAS indicates that exact $p$-values are only computed, by default, for samples of $n<$ 20 , and that asymptotic $p$-values are otherwise computed with an adjustment for ties only; however, the $p$-value generated appears to be the exact $p$-value, as it is equal to the exact $p$-value generated from Stata, and greater than the asymptotic $p$-value obtained through hand-calculation by a difference of approximately .0014 . Additionally, the continuity-corrected $p$-value provided 
by $R$ for the Wilcoxon Signed-Ranks test was greater than that which was produced through hand-calculation by a difference of approximately .0012. While no computational errors were present in the results of the Small $N$ analyses, it is of note that the "exact" $p$-value generated by SPSS for the Wilcoxon Mann-Whitney U test is misleading and more appropriately described as a "pseudo-exact" result (Bergmann et al., 2000). Specifically, SPSS uses Dineen and Blakesley's (1973) algorithm to compute the exact $p$-value, which has previously been shown to be invalid in the presence of ties, because the algorithm relies on the relative size of the two groups and does not consider the number of tied values (Bergmann et al., 2000; Lehmann, 1998). The formula used to compute the exact $p$-value in Stata, however, is exact and holds both when ties are present and when ties are absent (Hill \& Peto, 1971; Wilcoxon, Katti, \& Wilcox, 1970). It is also important to note that exact $p$-values are computed by default in $R$ for both Wilcoxon tests when sample sizes are small $(n<50)$; however, this only occurs when there are no ties present.

Statistical output. The output produced for each test statistic differed widely across the four statistical packages compared. Each package produces a variety of statistical output in addition to the specific test statistics that are typically required to be reported and interpreted in the literature, and these are described in detail in the preceding results sections for each statistical procedure. With regard to the inferential statistics that are necessary for one to make basic conclusions about the hypothesis that was tested (i.e., the test statistics, degrees of freedom, and $p$-values), several inconsistencies remained to be seen across SPSS, SAS, Stata, and $R$. While test statistics, degrees of freedom, and $p$-values were reported by all packages for Pearson's chisquare and Kruskal-Wallis tests, only SPSS provided these both with and without continuity correction for Pearson's chi-square, and only Stata provided these both with and without an adjustment for ties for the Kruskal-Wallis test. In the output produced for the Wilcoxon Mann- 
Whitney U test, SPSS alone generated Wilcoxon's $W$, Mann-Whitney's U, the asymptotic test statistic ( $z$-statistic), and the asymptotic $p$-value. In contrast, the output from SAS and Stata did not include the Mann-Whitney $U$ statistic, and while Wilcoxon's $W$ technically appears in the rank sum frequency table, it is neither clearly indicated nor differentiated from the other rank sum values reported in Stata's output. Additionally, only the Mann-Whitney U statistic and the continuity-corrected $p$-value are provided in the output generated in $R$. Finally, none of the four packages reported all of the fundamental inferential statistics in their output for the Wilcoxon Signed-ranks test. While both SPSS and Stata report the asymptotic $z$-statistic and $p$-value, neither report the Wilcoxon $T$ statistic, and while the Wilcoxon $T$ statistic and $p$-values are reported in the output of both SAS and $R$, neither of these packages report the asymptotic (SAS) or continuity corrected $(R) z$-statistic. Furthermore, it is important to note that SAS labels all test statistics as "S", regardless of the fact that several test statistics are commonly defined by specific labels, and incorrect labels were used in the output provided by $R$ for the Mann-Whitney U statistic (labeled as "W") and the Wilcoxon $T$ statistic (labeled as "V").

In addition to the differences in statistical output described above for the Large $N$ analyses, two differences were present in the statistical output for the Medium $N$ analyses, where exact $p$-values were reported, in addition to asymptotic $p$-values, for the Wilcoxon MannWhitney U and Wilcoxon Signed-Ranks test when conducted in Stata. These were also present in the statistical output generated in Stata for the Small $N$ analyses; however, an exact $p$-value was also reported, in addition to the asymptotic $p$-value, in the output of the Wilcoxon MannWhitney U test when conducted on the Small $N$ dataset in SPSS. Each exact $p$-values generated from Stata and SPSS was clearly differentiated from the asymptotic $p$-value in the statistical output. 


\section{Discussion}

In the current era of psychological science, much of the field's attention has been directed at methodological practices and reproducible science. Unfortunately, we have seen little evidence that this increased attention has brought any awareness to inconsistencies between statistical software packages. The present study aimed to determine whether the same results would be generated from the same non-parametric statistical procedures when performed on the same dataset by different statistical software packages, and our findings bring into question the extent to which reporting error and/or misinterpreted results are present in the psychological science literature. While we expected some minor discrepancies to exist between the computational procedures used by SPSS, SAS, Stata, and $R$, we were overwhelmed by our discovery of such pervasive inconsistency across the results provided by these packages. Of utmost concern to us is that these crucial discrepancies would have been so easily overlooked, had we not investigated them directly.

Overall, we have made a few conclusions from the present investigation. First, we conclude that the results of several common nonparametric tests and measures of normality differ when performed on the same data across SPSS, SAS, Stata, and $R$. These differences were primarily driven by the inconsistent use of default algorithmic procedures across software packages. These inconsistencies were supported by our supplementary analyses, which found that as sample size decreased, the more differences in statistical output appeared, which were primarily due to the automatic generation of exact $p$-values; however, the algorithms used to calculate exact $p$-values differed between packages. Second, we found that these differences also arise from computational and reporting errors. As sample size decreased, fewer computational errors were seen in the generated results. Although it is possible that algorithmic variation 
underlies these instances, this cannot be confirmed due to inaccurate or inadequate documentation provided by the software package. Third, we found that some statistical output generated from these nonparametric tests included unnecessary information. We believe that programs producing unnecessary and ambiguous output, especially without clearly indicating and describing produced values, may increase confusion and/or selective reporting of misleading results in the literature. In particular, we argue that confusion is more likely when a student, scientific researcher, or other non-statistician investigator attempts to interpret the results of a single test when multiple $p$-values are present in the generated output. This may be especially relevant when algorithms used to generate the different $p$-values are not clearly indicated in the output and/or defined in the documentation. At worst, when multiple $p$-values are provided for a single test, we fear that publication bias and the current "publish or perish" mentality in scientific research may lead to selective reporting of whichever $p$-value most closely supports the hypothesis. Taken together, these factors and discrepancies may contribute to the replication failure in the social and behavioral science literature.

\section{Limitations}

The current investigation has noteworthy limitations. Although we found differences in the results generated across software packages, we want to encourage caution when generalizing these findings to other programs and other versions of the software. Software developers (especially those working on open-access software like $R$ ) are continually updating their programs and correcting bugs in the software. Many of the errors we found may be due to bugs in the software that have not yet been discovered; thus, the findings presently discussed are specific to the programs we compared and the versions of these programs we used for the present analyses. We encourage readers to consider the context in which the present discrepancies across 
results were found and to use the present findings as motivation to more closely consider their own statistical results. Furthermore, our results may not generalize to other, more commonly used statistical procedures, such as parametric analyses. A large contributor to the heterogeneity we found in results across statistical packages was due to algorithmic variation, particularly those related to continuity corrections, ties, and the calculation of exact $p$-values. These procedures are not relevant in parametric analyses; thus, there may be fewer ways in which the results of parametric statistics could differ across software packages.

\section{Implications and Recommendations}

The present results raise questions about previous replication efforts. It is well known within the social and behavioral sciences that many landmark studies have failed to replicate. It is possible that some of these failures are explained by algorithmic variation or other differences in the computation or reporting of results between statistical software packages, and future replication efforts should be aware of this possibility and focus on determining whether failures to replicate may be, at least in part, due to differences between the software used. The heterogeneity we have demonstrated in our results speaks to the importance of open-access science; without knowing the underlying procedures used to generate statistical results, it may be difficult to directly replicate previous studies. Replication efforts within the social and behavioral sciences have made significant strides toward promoting open-access science, but work remains to be done.

We advocate for fully open-access science, including publicly hosting datasets, code/syntax used to generate results, and all study materials used in the final analysis. New organizations, such as the Open Science Foundation (OSF), have made considerable progress in the promotion of open-access science by emphasizing the pre-registration of scientific studies, 
where researchers may report all study procedures prior to beginning data collection and publish the results upon study completion. Additionally, OSF has data hosting options that allow researchers to post their datasets and analysis pipelines for future replication efforts. This level of transparency in science remains promising as more publishers require the documentation of study procedures and as academia begins to encourage pre-registration and sharing of all study data and materials. We suggest future studies continue this trend of transparency.

We strongly encourage all researchers, regardless of career stage, to carefully examine the documentation in software packages, and to assure the package selected is performing the correct test. Furthermore, it is necessary that the users are aware of the computational idiosyncrasies of the software used to conduct the statistical analyses, and it is recommended that the results are compared across platforms to avoid reporting errors or misleading results that are due to algorithmic variation, computational error, or other output-related characteristics of a given program. Of utmost importance is the necessity for adequate reporting of the statistical procedures used when analyzing data and full transparency when publishing the results of such analyses.

Some of the burden of open-access science lies on the shoulders of the software developers. Several issues were present in the help files and documentation provided by the software packages, where the information provided for the various analyses we conducted was often lacking sufficient information about a given test (e.g., $R$ ) or about the algorithms used to produce the results (e.g., Stata), and in some cases, the documentation was not easily accessible (e.g., SAS) or difficult to interpret (e.g., SPSS). Ambiguity in documentation could play a role in the inconsistent replication of scientific experiments that has been noted in recent years (OSF, 
2012), and it is essential that the software developers are made aware of the importance of clear, exhaustive, and easily accessible documentation for these packages.

\section{Conclusion}

The results of our study demonstrate the unreliability of results produced for nonparametric tests and measures of normality across SPSS, SAS, Stata, and $R$. The inconsistent results were primarily due to differences in the default algorithms used, however computational error and differences in statistical output also contributed to the unreliability of results. These discrepancies, along with unnecessary and/or unclearly defined statistical output generated for a given test are likely contributors to confusion, selective reporting or reporting error, and ultimately, replication failure. We urge researchers to refer to documentation when interpreting results (when it is available), compare their statistical results across software platforms, and describe all statistical procedures, including the statistical software package used, when publishing results in order to aid in the success future replication efforts.

\section{Author contributions}

C. B. Hodges is responsible for the conception of this project. C. B. Hodges, H. M. Lindsey, and B. Stone contributed equally to the design of the study and wrote the first draft of the manuscript. All authors participated in the data selection, statistical analyses, and documentation procedures, and all authors contributed to the revision, review, and approval of the final manuscript.

\section{Declaration of Conflicting Interests}

The authors declared no conflicts of interest with respect to the authorship or the publication of this article.

\section{Acknowledgments}


The authors would like to thank Logan Kowallis and Elizabeth Hovenden for their contribution to the preparation of this manuscript.

\section{Funding}

The authors have no funding to declare.

\section{Supplemental Material}




\section{References}

Aiken, L. S., West, S. G., \& Millsap, R. E. (2008). Doctoral training in statistics, measurement, and methodology in psychology: Replication and extension of Aiken, West, Sechrest, and Reno's (1990) survey of PhD programs in North America. American Psychologist, 63(1), 32.

Aiken, L. S., West, S. G., \& Millsap, R. E. (2009). Improving training in methodology enriches the science of psychology. American Psychologist, 64(1), 51-56. https://doi.org/10.1037/a0014161

Alder, A. G., \& Vollick, D. (2000). Undergraduate statistics in psychology: A survey of canadian institutions. Canadian Psychology/Psychologie canadienne, 41(3), 149-151.

Bakker, M., \& Wicherts, J. M. (2011). The (mis)reporting of statistical results in psychology journals. Behavioral Research Methods, 43(3), 666-678. https://doi.org/10.3758/s13428011-0089-5

Bauer, D. F. (1972). Constructing confidence sets using rank statistics. Journal of the American Statistical Association, 67, 687-690. https://doi.org/10.1080/01621459.1972.10481279

Bergmann, R., Ludbrook, J., \& Spooren, W. P. J. M. (2000). Different outcomes of the Wilcoxon-Mann-Whitney test from different statistics packages. The American Statistician, 54(1), 72-77. https://doi.org/10.1080/00031305.2000.10474513

Berle, D., \& Starcevic, V. (2007). Inconsistencies between reported test statistics and $p$-values in two psychiatry journals. International Journal of Methods in Psychiatric Research, 16(4), 202-207. https://doi.org/10.1002/mpr.225

Bliss, C. I. (1967). Statistics in biology (Vol. 1). New York, NY: McGraw-Hill. 
Bock, R. D. (1975). Multivariate statistical methods in behavioral research. New York, NY: McGraw-Hill.

Borghi, J. A., \& Van Gulick, A. E. (2018). Data management and sharing in neuroimaging: Practices and perceptions of MRI researchers. PLoS One, 13(7), e0200562. https://doi.org/10.1371/journal.pone.0200562

Borsboom, D. (2006). The attack of the psychometricians. Psychometrika, 71, 425-440.

Brown, B. L., Hendrix, S. B., Hedges, D. W., \& Smith, T. B. (2012). Multivariate analysis for the biobehavioral and social sciences: A graphical approach. Hobokan, NJ: John Wiley \& Sons.

Chambers, C. (2019). The seven deadly sins of psychology: A manifesto for reforming the culture of scientific practice. Princeton, NJ: Princeton University Press.

Conover, W. J. (1974). Some reasons for not using the yates continuity correction on $2 \times 2$ contingency tables. Journal of the American Statistical Association, 69(346), 374-376.

Cook, T. D., \& Campbell, D. T. (1979). Quasi-experimentation: Design and analysis issues for field settings. Boston, MA: Houghton Mifflin.

Cramér, H. (1946). Mathematical methods of statistics. Princeton, NJ: Princeton University Press.

Cureton, E. E. (1967). The normal approximation to the signed-rank sampling distribution when zero differences are present. Journal of the American Statistical Association, 62(319), 1068-1069.

Dar, R., Serlin, R. C., \& Omer, H. (1994). Misuse of statistical test in three decades of psychotherapy research. Journal of Consulting and Clinical Psychology, 62(1), 75-82. https://doi.org/10.1037//0022-006x.62.1.75 
Dineen, L. C., \& Blakesley, B. C. (1973). Algorithm AS 62: Generator for the sampling distribution of the Mann-Whitney U statistic. Applied Statistics, 22, 269-273.

Epskamp, S. (2019). Reproducibility and replicability in a fast-paced methodological world. Advances in Methods and Practices in Psychological Science, 2(2), 145-155.

Erceg-Hurn, D. M., \& Mirosevich, V. M. (2008). Modern robust statistical methods: An easy way to maximize the accuracy and power of your research. American Psychologist, 63(7), 591-601. https://doi.org/10.1037/0003-066X.63.7.591

Fisher, R. A. (1935). The design of experiments. Edinburgh: Oliver \& Boyd.

Friedrich, J., Buday, E., \& Kerr, D. (2000). Statistical training in psychology: A national survey and commentary on undergraduate programs. Teaching of Psychology, 27, 248-257.

Friedrich, J., Childress, J., \& Cheng, D. (2018). Replicating a national survey on statistical training in undergraduate psychology programs: Are there "new statistics" in the new millennium? Teaching of Psychology, 45(4), 312-323. https://doi.org/10.1177/0098628318796414

Garcia-Perez, M. A. (2012). Statistical conclusion validity: Some common threats and simple remedies. Frontiers in Psychology, 3, 325. https://doi.org/10.3389/fpsyg.2012.00325

Gelman, A., \& O'Rourke, K. (2014). Difficulties in making inferences about scientific truth from distributions of published $p$-values. Biostatistics, 15(1), 18-23; Discussion 39-45. https://doi.org/10.1093/biostatistics/kxt034

Gibbons, J. D., \& Chakraborti, S. (2011). Nonparametric statistical inference (5th ed.). Boca Raton, FL: Taylor \& Francis Group. 
Giner-Sorolla, R. (2012). Science or art? How aesthetic standards grease the way through the publication bottleneck but undermine science. Perspectives on Psychological Science, 7(6), 562-571. https://doi.org/10.1177/1745691612457576

Haber, M. (1982). The continuity correction and statistical testing. International Statistical Review, 50, 135-144.

Head, M. L., Holman, L., Lanfear, R., Kahn, A. T., \& Jennions, M. D. (2015). The extent and consequences of $p$-hacking in science. PLoS Biology, 13(3), e1002106. https://doi.org/10.1371/journal.pbio.1002106

Hill, I. D., \& Peto, R. (1971). Algorithm AS 35: Probabilities derived from finite populations. Applied Statistics, 20, 99-105.

Hitchcock, D. B. (2009). Yates and contingency tables: 75 years later. Electronic Journal for History of Probability and Statistics, 5, 1-14.

Hoekstra, R., Kiers, H. A., \& Johnson, A. (2012). Are assumptions of well-known statistical techniques checked, and why (not)? Frontiers in Psychology, 3, 137. https://doi.org/10.3389/fpsyg.2012.00137

Hollander, M., Wolfe, D. A., \& Chicken, E. (2014). Nonparametric statistical methods. ( $3^{\text {rd }}$ ed.). Hoboken, NJ: John Wiley \& Sons.

Huang, Y., \& Bentler, P. M. (2015). Behavior of asymptotically distribution free test statistics in covariance versus correlation structure analysis. Structural Equation Modeling: A Multidisciplinary Journal, 22(4), 489-503. https://doi.org/10.1080/10705511.2014.954078 
Iman, R. L. (1974). Use of a $t$-statistic as an approximation to the exact distribution of Wilcoxon Signed Ranks test statistic. Communications in Statistics-Simulation and Computation, 3, 795-806.

Ioannidis, J. P. (2005). Why most published research findings are false. PLoS Med, 2(8), e124. https://doi.org/10.1371/journal.pmed.0020124

Ioannidis, J. P. (2014). How to make more published research true. PLoS Medicine, 11(10), e1001747. https://doi.org/10.1371/journal.pmed.1001747

Joanes, D. N., \& Gill, C. A. (1998). Comparing measures of sample skewness and kurtosis. The Statistician, 47, 183-189.

Keeling, K. B., \& Pavur, R. J. (2007). A comparative study of the reliability of nine statistical software packages. Computational Statistics \& Data Analysis, 51(8), 3811-3831.

Kerr, N. L. (1998). HARKing: Hypothesizing after the results are known. Personality and Social Psychology Review, 2(3), 196-217.

Keselman, H. J., Huberty, C. J., Lix, L. M., Olejnik, S., Cribbie, R. A., Donahue, B., .. . Levin, J. R. (1998). Statistical practices of educational researchers: An analysis of their ANOVA, MANOVA, and ANCOVA analyses. Review of Educational Research, 68(3), $350-386$.

Köhler, T., Landis, R. S., \& Cortina, J. M. (2017). From the editors: Establishing methodological rigor in quantitative management learning and education research: The role of design, statistical methods, and reporting standards. Academy of Management Learning and Education, 16(2), 173-192. https://doi.org/10.5465/amle.2017.0079

Lane, K. A., Banaji, M. B., Nosek, B. A., \& Greenwald, A. G. (2007). Understanding and using the Implicit Association Test: What we know (so far) about the method. In B. 
Wittenbrink \& N. Schwarz (Eds.), Implicit measures of attitudes (pp. 59-102). New York, NY: Guilford Press.

Lehmann, E. L. (1998). Nonparametrics: Statistical methods based on ranks (Revised $1^{\text {st }}$ ed.). Upper Saddle River, NJ: Prentice Hall.

Lehmann, E. L., \& D’Abrera, H. J. M. (2006). Nonparametrics: Statistical methods based on ranks (Revised ed.). San Francisco, CA: Holden-Day.

Levine, T. R., \& Atkin, C. (2004). The accurate reporting of software-generated $p$-values: A cautionary research note. Communication Research Reports, 21(3), 324-327. https://doi.org/10.1080/08824090409359995

Mantel, N. (1976). The continuity correction. The American Statistician, 30, 103-104.

Maxwell, E. A. (1976). Analysis of contingency tables and further reasons for not using Yates correction in 2×2 tables. Canadian Journal of Statistics 4, 277-290.

McCoach, D. B., Rifenbark, G. G., Newton, S. D., Li, X., Kooken, J., Yomtov, D., . . Bellara, A. (2018). Does the package matter? A comparison of five common multilevel modeling software packages. Journal of Educational and Behavioral Statistics, 43(5), 594-627.

McCullough, B. (2000). Is it safe to assume that software is accurate? International Journal of Forecasting, 16(3), 349-357.

McCullough, B. D., \& Heiser, D. A. (2008). On the accuracy of statistical procedures in Microsoft Excel 2007. Computational Statistics \& Data Analysis, 52(10), 4570-4578.

McNemar, Q. (1947). Note on the sampling error of the difference between correlated proportions or percentages. Psychometrika, 12, 153-157. 
Munafò, M. R., Nosek, B. A., Bishop, D. V., Button, K. S., Chambers, C. D., Du Sert, N. P., . . Ioannidis, J. P. (2017). A manifesto for reproducible science. Nature human behaviour, 1(1), 0021.

Neave, H. R., \& Worthington, P. L. (1988). Distribution-free tests. London, UK: Unwin Hyman Ltd.

Nieuwenhuis, S., Forstmann, B. U., \& Wagenmakers, E.-J. (2011). Erroneous analysis of interactions in neuroscience: A problem of significance. Nature Neuroscience, 14, 11051107.

Open Science Collaboration. (2012). An open, large-scale, collaborative effort to estimate the reproducibility of psychological science. Perspectives on Psychological Science, 7(6), 657-660.

Open Science Collaboration. (2015). Estimating the reproducibility of psychological science. Science, 349(6251), aac4716. https://doi.org/10.1126/science/aac4716

Osborne, J. (2008). Sweating the small stuff in educational psychology: How effect size and power reporting failed to change from 1969 to 1999, and what that means for the future of changing practices. Educational Psychology, 28, 151-160.

Osborne, J., \& Waters, E. (2002). Four assumptions of multiple regression that researchers should always test. Practical Assessment, Research \& Evaluation, 8(2), 1-9.

Oster, R. A., \& Hilbe, J. M. (2008a). An examination of statistical software packages for parametric and nonparametric data analyses using exact methods. The American Statistician, 62(1), 74-84. https://doi.org/10.1198/000313008X268955 
Oster, R. A., \& Hilbe, J. M. (2008b). Rejoinder to "An examination of statistical software packages for parametric and nonparametric data analyses using exact methods". The American Statistician, 62(2), 173-176. https://doi.org/10.1198/000313008X306853

Potvin, C., \& Roff, D. A. (1993). Distribution-free and robust statistical methods: Viable alternative to parametric statistics? Ecology, 74(6), 1617-1628.

Pratt, J. W. (1959). Remarks on zeros and ries in the Wilcoxon Signed Rank procedures. Journal of the American Statistical Association, 54, 655-667.

Rubin, J. D., Bell, S., \& McClelland, S. I. (2018). Graduate education in qualitative methods in US psychology: Current trends and recommendations for the future. Qualitative Research in Psychology, 15(1), 29-50.

Schatz, P., Jay, K. A., McComb, J., \& McLaughlin, J. R. (2005). Misuse of statistical tests in Archives of Clinical Neuropsychology publications. Archives of Clinical Neuropsychology, 20(8), 1053-1059. https://doi.org/10.1016/j.acn.2005.06.006

Shrout, P. E., \& Rodgers, J. L. (2018). Psychology, science, and knowledge construction: Broadening perspectives from the replication crisis. Annual Review of Psychology, 69, 487-510. https://doi.org/10.1146/annurev-psych-122216-011845

Siegel, S. (1956). Nonparametric statistics for the behavioral sciences. New york: Mcgraw-hill. New York, NY: McGraw-Hill.

Siegel, S. (1957). Nonparametric statistics. The American Statistician, 11(3), 13-19.

Siegel, S., \& Castellan, N. J. (1988). Non-parametric statistics for the behavioural sciences $\left(2^{\text {nd }}\right.$ ed.). New York, NY: McGraw-Hill. 
Simmons, J. P., Nelson, L. D., \& Simonsohn, U. (2011). False-positive psychology: Undisclosed flexibility in data collection and analysis allows presenting anything as significant. Psychological Science, 22(11), 1359-1366. https://doi.org/10.1177/0956797611417632

Simonsohn, U., Nelson, L. D., \& Simmons, J. P. (2014). P-curve: A key to the file-drawer. Journal of Experimental Psychology: General, 143(2), 534-547. https://doi.org/10.1037/a0033242.supp

Stefanescu, C., Berger, V. W., \& Hershberger, S. (2005). Yates's continuity correction. In B. S. Everit \& D. Howell (Eds.), Encyclopedia of Statistics in Behavioural Science (Vol. 4, pp. 2127-2129). Hoboken, NJ: John Wiley \& Sons.

Wang, J., \& Johnson, D. E. (2019). An examination of discrepancies in multiple imputation procedures between SAS ${ }^{\circledR}$ and SPSS ${ }^{\circledR}$. The American Statistician, 73(1), 80-88. https://doi.org/10.1080/00031305.2018.1437078

Wasserman, L. (2006). All of nonparametric statistics. New York, NY: Springer.

Whitley, E., \& Ball, J. (2002). Statistics review 6: Nonparametric methods. Critical Care, 6, 509-513. https://doi.org/10.1186/cc1820

Wicherts, J. M., Veldkamp, C. L. S., Augusteijn, H. E. M., Bakker, M., van Aert, R. C. M., \& van Assen, M. A. L. M. (2016). Degrees of freedom in planning, running, analyzing, and reporting psychological studies: A checklist to avoid p-hacking. Frontiers in Psychology, 7(1832). https://doi.org/10.3389/fpsyg.2016.01832

Wilcoxon, F. (1945). Individual comparisons by ranking methods. Biometrics Bulletin, 1(6), 8083.

Wilcoxon, F., Katti, S. K., \& Wilcox, R. A. (1970). Critical values and probability levels for the Wilcoxon Rank Sum test and the Wilcoxon Signed Rank test. In The Institute of 


\author{
Mathematical Statistics, H. L. Harter, \& D. B. Owen (Eds.), Selected tables in \\ mathematical statistics ( $1^{\text {st }}$ ed., Vol. 1, pp. 171-259). Providence, RI: American \\ Mathematical Society.
}

Xu, K., Nosek, B., \& Greenwald, A. (2014). Psychology data from the race implicit association test on the Project Implicit demo website. Journal of Open Psychology Data, 2(1).

Zimmerman, D. W. (1998). Invalidation of parametric and nonparametric statistical tests by concurrent violation of two assumptions. Journal of Experimental Education, 67, 55-68.

Zimmerman, D. W. (2000). Statistical significance levels of nonparametric tests biased by heterogeneous variances of treatment groups. Journal of General Psychology, 127, 354364.

Zimmerman, D. W., \& Zumbo, B. D. (1993). The relative power of parametric and nonparametric statistical methods. In G. Keren \& C. Lewis (Eds.), A handbook for data analysis in the behavioral sciences: Methodological issues (pp. 481-518). Mahwah, NJ: Lawrence Erlbaum Associates. 
Table 1

Description of variables selected or generated from the Race IAT Public 2018 dataset

\begin{tabular}{|c|c|c|c|c|}
\hline $\begin{array}{l}\text { Variable Name } \\
\text { (IAT name) }\end{array}$ & Description & $\begin{array}{l}\text { Measurement } \\
\text { Scale }\end{array}$ & Levels & $N$ \\
\hline $\begin{array}{l}\text { id } \\
\text { (session_ID) }\end{array}$ & $\begin{array}{l}\text { Unique session identification number } \\
\text { generated when one begins the IAT }\end{array}$ & String & - & 414,167 \\
\hline $\begin{array}{l}\text { sex } \\
\text { (birthsex) }\end{array}$ & Biological sex assigned at birth & Nominal & 2 & 394,421 \\
\hline $\begin{array}{l}\text { race } \\
\text { (raceomb_002) }\end{array}$ & Racial association & Nominal & 8 & 360,660 \\
\hline $\begin{array}{l}\text { black } \\
\text { (tblack_Oto10) }\end{array}$ & $\begin{array}{l}\text { Feelings of warmth or coldness } \\
\text { toward Black people }\end{array}$ & Ordinal & 11 & 393,274 \\
\hline $\begin{array}{l}\text { white } \\
\text { (twhite_Oto10) }\end{array}$ & $\begin{array}{l}\text { Feelings of warmth or coldness } \\
\text { toward White people }\end{array}$ & Ordinal & 11 & 393,201 \\
\hline $\begin{array}{l}\text { order } \\
\text { (Order) }\end{array}$ & Task presentation order & Nominal & 2 & 414,167 \\
\hline $\begin{array}{l}\text { latency } \\
\text { (Mn_Rt_all_3467) }\end{array}$ & $\begin{array}{l}\text { Mean reaction time across all task } \\
\text { blocks }\end{array}$ & Ratio & - & 414,167 \\
\hline $\begin{array}{l}\text { discrim1 } \\
\text { (D_biep.White_Good_36) }\end{array}$ & $\begin{array}{l}\text { Discriminability score on blocks } 3 \\
\text { and } 6\end{array}$ & Interval & - & 414,167 \\
\hline $\begin{array}{l}\text { discrim2 } \\
\text { (D_biep.White_Good_47) }\end{array}$ & $\begin{array}{l}\text { Discriminability score on blocks } 4 \\
\text { and } 7\end{array}$ & Interval & - & 414,166 \\
\hline
\end{tabular}

Note. The full codebook for the variables used in the present study can be accessed in our OSF storage directory at https://osf.io/35umb/ 
Table 2

Descriptions of the nonparametric tests included in the present analyses

\begin{tabular}{|c|c|c|c|}
\hline Analysis & Description & Null Hypothesis & $\begin{array}{c}\text { Parametric } \\
\text { equivalent }\end{array}$ \\
\hline $\begin{array}{l}\text { Pearson's Chi- } \\
\text { square }\end{array}$ & $\begin{array}{l}\text { A test used to determine } \\
\text { if a relationship exists } \\
\text { between discrete, } \\
\text { categorical variables. }\end{array}$ & $\begin{array}{l}\text { The variables are } \\
\text { independent of (not } \\
\text { associated with) one } \\
\text { another. }\end{array}$ & None \\
\hline Spearman's rho & $\begin{array}{l}\text { A test used to measure } \\
\text { the degree of association } \\
\text { between two ordinal, or } \\
\text { rank-ordered variables. }\end{array}$ & $\begin{array}{l}\text { There is no monotonic } \\
\text { relationship (correlation) } \\
\text { between the variables for } \\
\text { the population. }\end{array}$ & $\begin{array}{l}\text { Pearson's product } \\
\text { moment correlation }\end{array}$ \\
\hline Kruskal-Wallis & $\begin{array}{l}\text { A rank-based test used } \\
\text { to assess the } \\
\text { independence of the } \\
\text { populations of two or } \\
\text { more independent } \\
\text { samples. }\end{array}$ & $\begin{array}{l}\text { The samples come from } \\
\text { populations with the same } \\
\text { location (i.e., medians and } \\
\text { quartiles coincide). }\end{array}$ & $\begin{array}{l}\text { One-way Analysis } \\
\text { of Variance }\end{array}$ \\
\hline $\begin{array}{l}\text { Wilcoxon-Mann- } \\
\text { Whitney U }\end{array}$ & $\begin{array}{l}\text { A rank-based test used } \\
\text { to assess the } \\
\text { independence of the } \\
\text { populations of two } \\
\text { independent samples. }\end{array}$ & $\begin{array}{l}\text { The samples come from } \\
\text { populations with the same } \\
\text { location (i.e., medians and } \\
\text { quartiles coincide). }\end{array}$ & $\begin{array}{l}\text { Independent } \\
\text { samples } \\
\text { (Student's) } t \text {-test }\end{array}$ \\
\hline $\begin{array}{l}\text { Wilcoxon Signed } \\
\text { Ranks test }\end{array}$ & $\begin{array}{l}\text { A rank-based test used } \\
\text { to assess the equality of } \\
\text { matched pairs of } \\
\text { observations. }\end{array}$ & $\begin{array}{l}\text { There is no systematic } \\
\text { tendency for the paired } \\
\text { differences to be positive } \\
\text { or negative. }\end{array}$ & $\begin{array}{l}\text { Paired/Matched } \\
\text { samples } t \text {-test }\end{array}$ \\
\hline
\end{tabular}


Table 3

Comparison of results obtained across statistical software platforms for a large sample $(N=414,167)$ with missing data

\begin{tabular}{|c|c|c|c|c|c|c|c|}
\hline Test & Algorithm & Statistic & Hand-calculation & SPSS & SAS & Stata & $R$ \\
\hline \multirow[t]{4}{*}{ Pearson's Chi-Square } & & $d f$ & 1 & 1 & 1 & 1 & 1 \\
\hline & Asymptotic & $x^{2}$ & 0.0103590146942365 & .010 & 0.010 & 0.0104 & - \\
\hline & & $p$ & 0.918931900249052 & .919 & 0.9189 & 0.919 & - \\
\hline & & $p$ & 0.9215456711388 & .922 & - & - & 0.9215 \\
\hline Spearman's rho & Ties & $r_{\mathrm{s}}$ & 0.584615783557598 & .585 & 0.5847 & 0.5847 & 0.5847216 \\
\hline \multirow[t]{5}{*}{ Kruskal-Wallis } & & $d f$ & 7 & 7 & 7 & 7 & 7 \\
\hline & Asymptotic & $x^{2}$ & 1049.5433535832 & - & 1049.5434 & 1049.517 & 1049.5 \\
\hline & & $p$ & $2.371243848 \mathrm{E}-222$ & - & $<.0001$ & 0.0001 & $<2.2 \mathrm{e}-16$ \\
\hline & Ties & $x^{2}$ & 1049.5433536029 & 1049.543 & - & 1049.517 & - \\
\hline & & $p$ & 2.371243824E-222 & .000 & - & 0.0001 & - \\
\hline \multirow[t]{3}{*}{ Wilcoxon Mann-Whitney U } & & $W$ & 38665285438 & $3.867 \mathrm{E}+10$ & $3867 e+10^{b}$ & - & - \\
\hline & Continuity & $z$ & 1.92010895022983 & - & 1.92011 & - & - \\
\hline & & $p$ & 0.0548441390032153 & - & 0.0548 & - & 0.05484 \\
\hline \multirow[t]{5}{*}{ Wilcoxon Signed-Ranks } & & $T$ & 36940509739.5 & - & $-5.9 e+9^{b}$ & - & $3.6941 \mathrm{e}+10^{\mathrm{d}}$ \\
\hline & Ties $^{a}$ & $z$ & -77.2380276272048 & -77.238 & - & 77.238 & - \\
\hline & & $p$ & $0.000000 \mathrm{E}+00$ & .000 & $<.0001$ & 0.0000 & - \\
\hline & Continuity & $z$ & -77.2380276337031 & - & - & - & - \\
\hline & & $p$ & $0.000000 \mathrm{E}+00$ & - & - & - & $<2.2 \mathrm{e}-16$ \\
\hline \multirow[t]{2}{*}{ Skewness } & Population & & 165.278818163546 & - & - & 165.2788 & - \\
\hline & Sample & & 165.279416761152 & 165.279 & 165.27942 & - & - \\
\hline Kurtosis & Population & & 39297.7761139629 & - & - & 39297.78 & - \\
\hline
\end{tabular}

Note. Results are organized according to the default algorithms used by each program, as noted in the respective output and/or help files/documentation, and all results are presented exactly as they are reported in the statistical output generated by each package. Bolded values indicate results that are inconsistent with the hand-calculated results. The values reported from "asymptotic" algorithms are those reported with no correction for ties or continuity applied. Dashes indicate values that were not provided in the default output by the respective statistical package.

${ }^{a}$ No ties present

bTest statistic labeled as "S"

cTest statistic labeled as "W"

dTest statistic labeled as "V" 
Table 4

Comparison of results obtained across statistical software platforms for a medium sample $(N=100)$ with no missing data

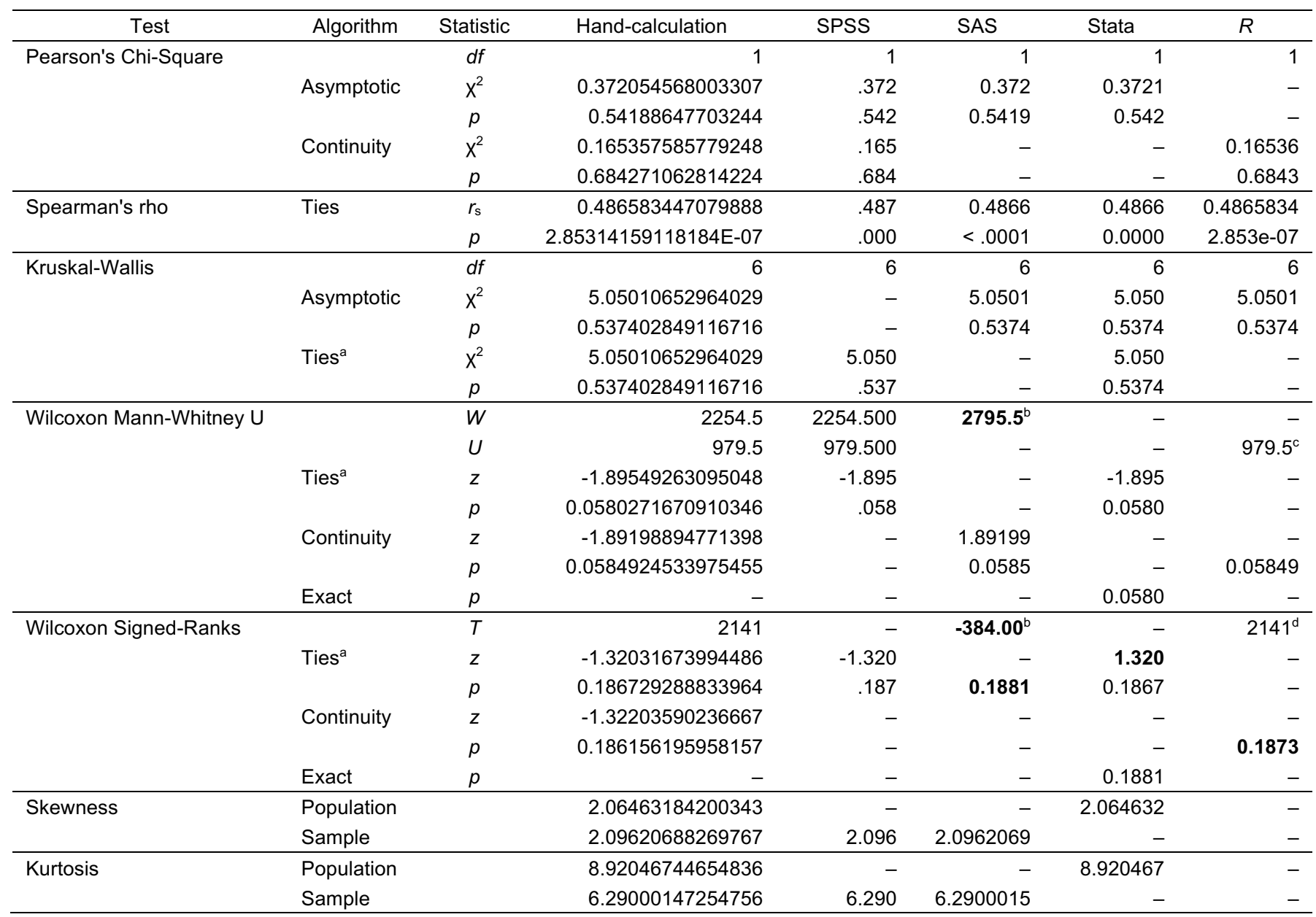

Note. Results are organized according to the default algorithms used by each program, as noted in the respective output and/or help files/documentation, and all results are presented exactly as they are reported in the statistical output generated by each package. Bolded values indicate results that are inconsistent with the hand-calculated results. The values reported from "asymptotic" algorithms are those reported with no correction for ties or continuity applied. Dashes indicate values that were not provided in the default output by the respective statistical package.

${ }^{a}$ No ties present

bTest statistic labeled as "S"

cTest statistic labeled as "W"

dTest statistic labeled as "V" 
Table 5

Comparison of results obtained across statistical software platforms for a small sample $(N=30)$ with no missing data

\begin{tabular}{|c|c|c|c|c|c|c|c|}
\hline Test & Algorithm & Statistic & Hand-calculation & SPSS & SAS & Stata & $R$ \\
\hline \multirow[t]{4}{*}{ Pearson's Chi-Square } & & $d f$ & 1 & 1 & 1 & 1 & 1 \\
\hline & Asymptotic & $x^{2}$ & 0.200892857142857 & .201 & 0.201 & 0.2009 & - \\
\hline & & $p$ & 0.654001120664955 & .654 & 0.6540 & 0.654 & - \\
\hline & & $p$ & 0.940451984965834 & .940 & - & - & 0.9405 \\
\hline Spearman's rho & Ties & $r_{\mathrm{s}}$ & 0.483532268795601 & .484 & 0.4835 & 0.4835 & 0.4835323 \\
\hline \multirow{4}{*}{ Kruskal-Wallis } & Asymptotic & $x^{2}$ & 0.320430107526875 & - & 0.3204 & 0.320 & 0.32043 \\
\hline & & $p$ & 0.851960551941017 & - & 0.8520 & 0.8520 & 0.852 \\
\hline & Ties $^{a}$ & $x^{2}$ & 0.320430107526875 & .320 & - & 0.320 & - \\
\hline & & $p$ & 0.851960551941017 & .852 & - & 0.8520 & - \\
\hline \multirow[t]{3}{*}{ Wilcoxon Mann-Whitney U } & & $w$ & 205 & 205.000 & $205^{b}$ & - & - \\
\hline & & $p$ & 0.626317808037939 & - & 0.6263 & - & 0.6263 \\
\hline & Exact & $p$ & - & .637 & - & 0.6319 & - \\
\hline \multirow[t]{6}{*}{ Wilcoxon Signed-Ranks } & & $T$ & 94 & - & $-138.5^{b}$ & - & $94^{d}$ \\
\hline & Ties $^{a}$ & $z$ & -2.84871500065283 & -2.849 & - & 2.849 & - \\
\hline & & $p$ & 0.00438961828903961 & .004 & 0.0027 & 0.0044 & - \\
\hline & Continuity & $z$ & -2.85899917033028 & - & - & - & - \\
\hline & & $p$ & 0.00424979896216319 & - & - & - & - \\
\hline & Exact & $p$ & - & - & - & 0.0035 & 0.003475 \\
\hline \multirow[t]{2}{*}{ Skewness } & Population & & 0.551479475721913 & - & - & 0.5514795 & - \\
\hline & Sample & & 0.580939556732467 & .581 & 0.5809396 & - & - \\
\hline
\end{tabular}

Note. Results are organized according to the default algorithms used by each program, as noted in the respective output and/or help files/documentation, and all results are presented exactly as they are reported in the statistical output generated by each package or across packages, in the case of exact $p$-values. Bolded values indicate results that are inconsistent with the hand-calculated results. The values reported from "asymptotic" algorithms are those reported with no correction for ties or continuity applied. Dashes indicate values that were not provided in the default output by the respective statistical package.

aNo ties present

bTest statistic labeled as "S"

cTest statistic labeled as "W"

dTest statistic labeled as "V" 


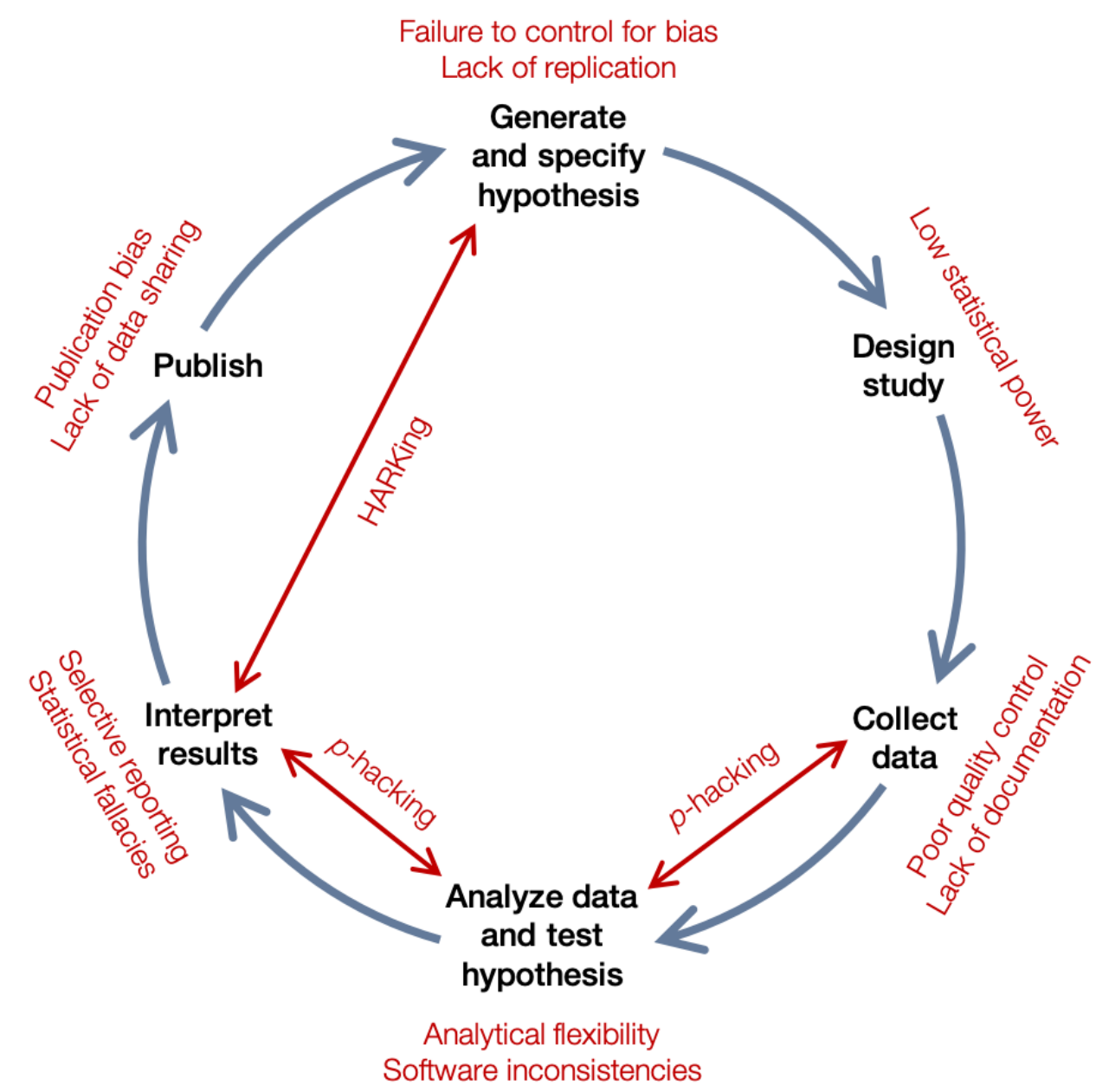

Figure 1. Questionable research practices (red, inside) and researcher degrees of freedom (red, outside) compromise the hypothetico-deductive model of the scientific method (blue/black). Questionable research practices include $p$-hacking, which manifests as collecting data until statistically significant effects are found and selectively reporting analyses with desirable outcomes, and hypothesizing after results are known (HARKing), which involves generating a hypothesis from the results of analyses and presenting it as a priori. There are several opportunities for researcher degrees of freedom to impede the scientific method including a failure to control for bias, lack of replication, low statistical power, poor quality control, lack of documentation, analytical flexibility, inconsistencies in algorithms used across statistical 
software (particularly relevant to the present study), selective reporting, statistical fallacies, publication bias, and lack of data sharing. These factors work together to undermine the robustness of published research. Adapted from Chambers (2019) and Munafò et al. (2017). 

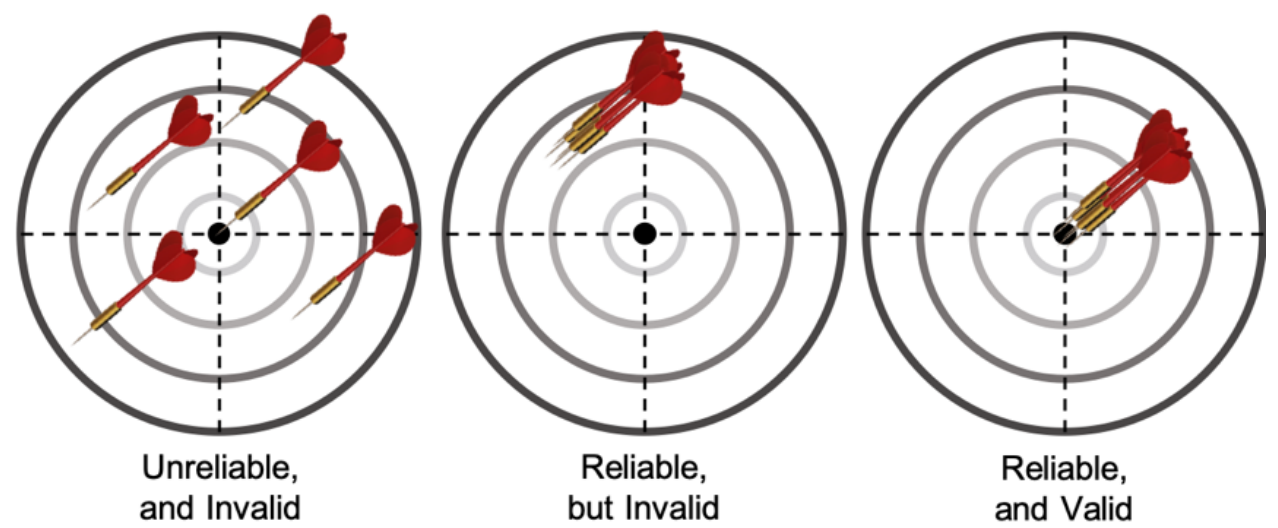

Figure 2. Reliability is necessary but not sufficient for validity. Validity is not present when results are unreliable (left) and may not be present when results are reliable but produced through the same, improper method (may be the result of direct replication; middle). Validity is only supported by reliable results that are obtained through adequate methods (right). 